\title{
A genome-wide scanning and fine mapping study of COGA data Hsin-Chou Yang ${ }^{1}$, Chien-Ching Chang ${ }^{1}$, Chin-Yu Lin ${ }^{2}$, Chun-Liang Chen ${ }^{1}$, Chin-Yu Lin ${ }^{1}$ and Cathy SJ Fann*1,2
}

\author{
Address: ${ }^{1}$ Institute of Biomedical Sciences, Academia Sinica, Taipei, Taiwan and ${ }^{2}$ Institute of Public Health, Yang-Ming University, Taipei, Taiwan \\ Email: Hsin-Chou Yang - hsinchou@ibms.sinica.edu.tw; Chien-Ching Chang - jenny@ibms.sinica.edu.tw; Chin- \\ YuLin-g39207013@ym.edu.tw; Chun-Liang Chen - kenny@ibms.sinica.edu.tw; Chin-Yu Lin - ta-a630050@mail.taipei.gov.tw; \\ Cathy SJ Fann* - csjfann@ibms.sinica.edu.tw \\ * Corresponding author
}

from Genetic Analysis Workshop 14: Microsatellite and single-nucleotide polymorphism

Noordwijkerhout, The Netherlands, 7-10 September 2004

Published: 30 December 2005

BMC Genetics 2005, 6(Suppl I):S30 doi:I0.II86/I47I-2I56-6-SI-S30

\begin{abstract}
A thorough genetic mapping study was performed to identify predisposing genes for alcoholism dependence using the Collaborative Study on the Genetics of Alcoholism (COGA) data. The procedure comprised whole-genome linkage and confirmation analyses, single locus and haplotype fine mapping analyses, and gene $x$ environment haplotype regression. Stratified analysis was considered to reduce the ethnic heterogeneity and simultaneously family-based and case-control study designs were applied to detect potential genetic signals. By using different methods and markers, we found high linkage signals at DIS225 (253.7 cM), DIS547 (279.2 cM), D2SI356 (64.6 $\mathrm{cM})$, and D7S2846 (56.8 cM) with nonparametric linkage scores of 3.92, 4.10, 4.44, and 3.55, respectively. We also conducted haplotype and odds ratio analyses, where the response was the dichotomous status of alcohol dependence, explanatory variables were the inferred individual haplotypes and the three statistically significant covariates were age, gender, and max drink (the maximum number of drinks consumed in a 24 -hr period). The final model identified important ADrelated haplotypes within a candidate region of $N R X N I$ at $2 \mathrm{p} 2 \mathrm{I}$ and a few others in the inter-gene regions. The relative magnitude of risks to the identified risky/protective haplotypes was elucidated.
\end{abstract}

\section{Background}

Alcohol dependence (AD) is a complex disorder accompanying familial aggregation and etiological heterogeneity. The development of $\mathrm{AD}$ involves genetic and environmental components as well as gene $\times$ gene and gene $x$ environmental interactions. Due to these factors, results from different studies often diverge [1].

Owing to the advancement of biotechnology, enormous numbers of short tandem repeat polymorphisms (STRPs) and single-nucleotide polymorphisms are available to help the process of gene mapping. In this report, STRP and SNP markers were integrated and a five-stage procedure was designed to identify the putative $\mathrm{AD}$ loci and to eluci- date the genotype-phenotype-covariate relationship. Different methodologies (linkage analysis, association fine mapping, haplotype inference, and regression model) were considered for statistical analyses, different populations (whole, non-Black, and non-White populations) for heterogeneity issues, different types of markers (STRPs and SNPs) for linkage mapping, different densities of SNPs (Illumina and Affymetrix) for association study, and different data structures (family data and case-control data) for study design to yield reliable conclusions. 


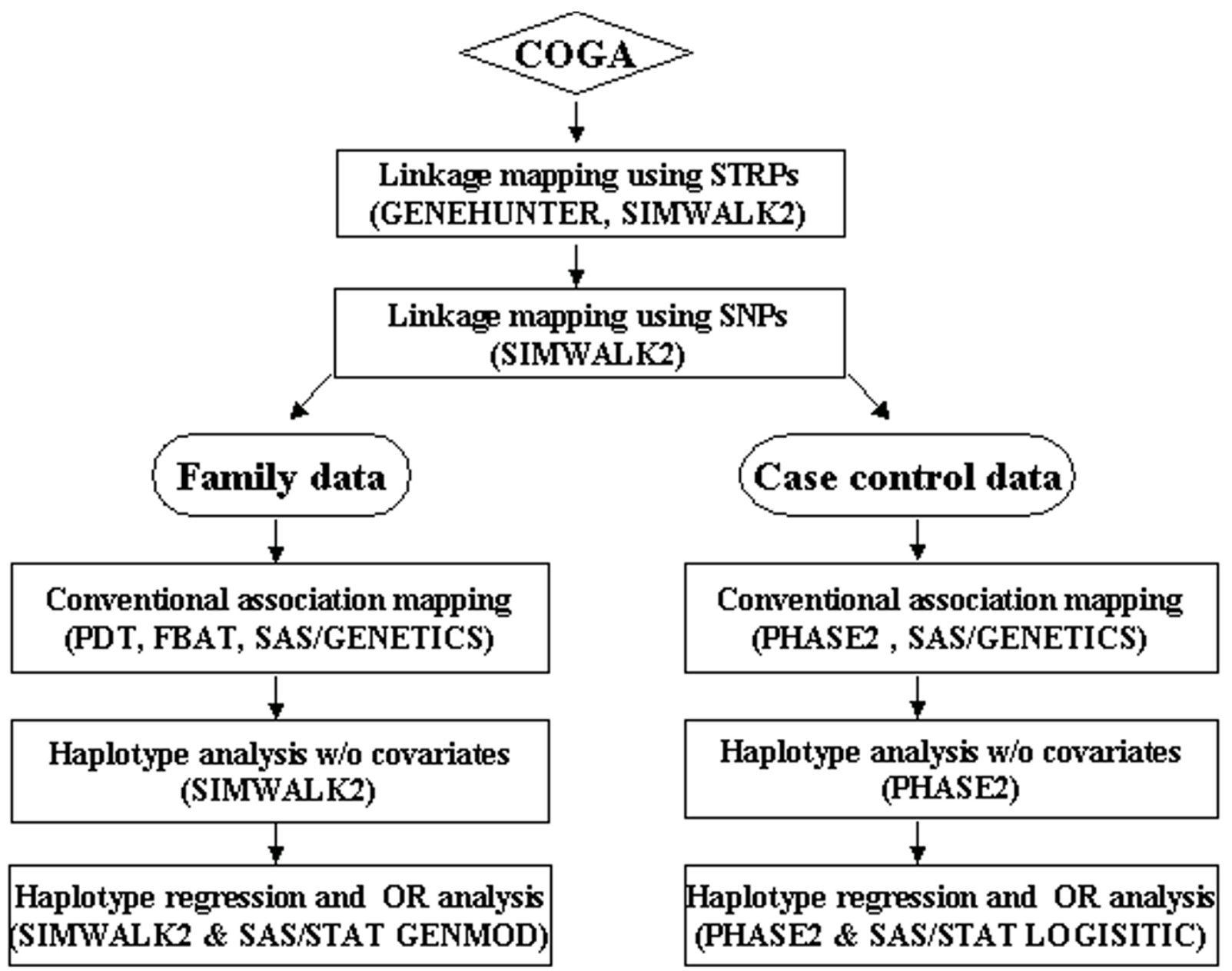

Figure I

The flow chart of statistical analyses.

\section{Methods}

Data description

From the COGA ascertainment criteria, the numbers of total patients, pure unaffected individuals, and others were 643 (39.84\%), 285 (17.66\%), and $686(42.50 \%)$, respectively. The category "others" was considered as "unknown" throughout our analyses. On average, 60\% of parents' genotypes were available.

In total, 315 STRPs, 4,720 Illumina SNPs, and 11,120 Affymetrix SNPs on the 22 autosomal chromosomes with average spacing of $11.53 \mathrm{cM}, 0.75 \mathrm{cM}$, and $0.32 \mathrm{cM}$ were considered. The genetic map was provided by the Genetic Analysis Workshop 14 (GAW14) working group.

Ethnic heterogeneity was considered by stratifying the studied families as pure "non-Black" and "non-White" families, i.e., families where none of the members were from the Black population and vice versa. The non-Black population contained 1,300 individuals from 119 families and non-White families contained 247 individuals from 19 families. Other families were not included in this report. In addition to family data, founders from each family were selected for case-control data that contained 

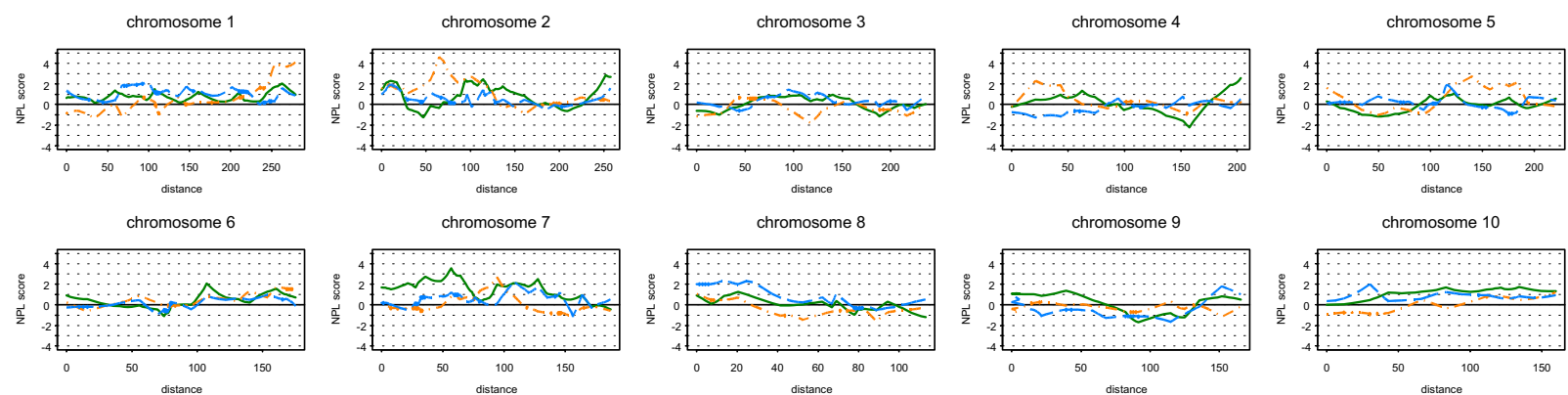

chromosome 11

chromosome 12
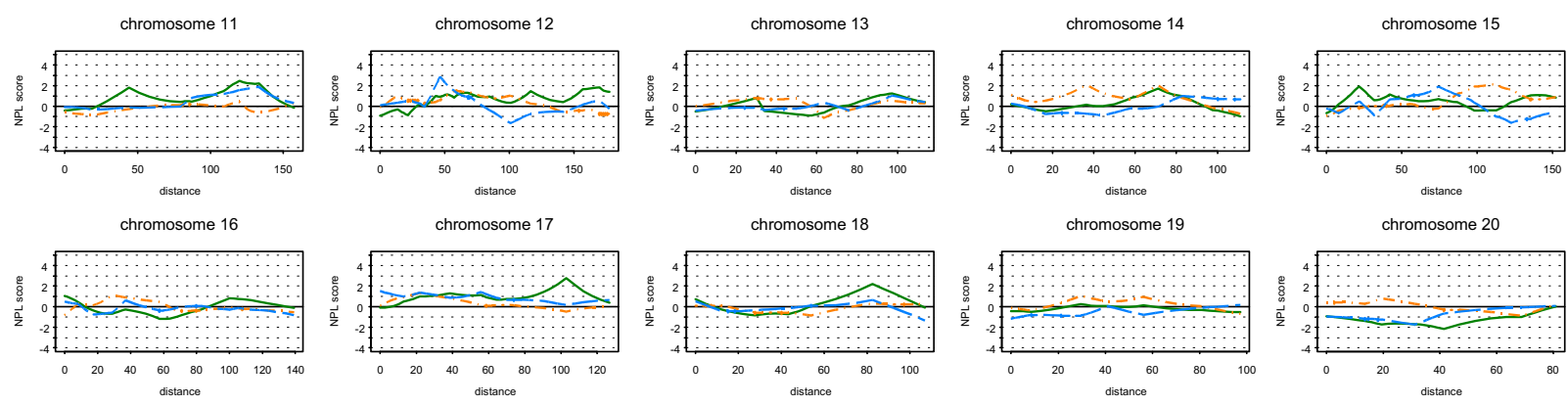

chromosome 21

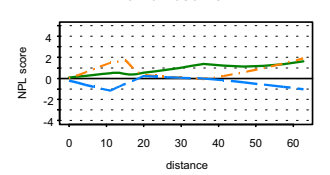

chromosome 22
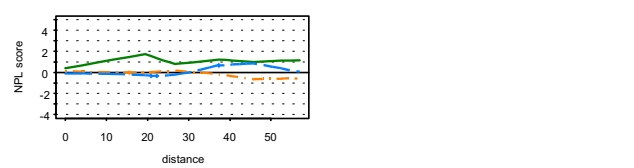

$\begin{array}{ll}\text { Whole } & \\ \text { Non-black } & -\cdots \cdot- \\ \text { Non-white } & -\ldots\end{array}$

SNP linkage in chromosome 1
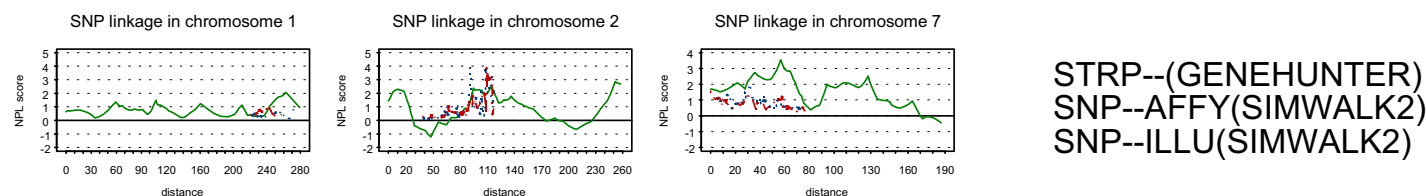

STRP--(GENEHUNTER)

SNP--ILLU(SIMWALK2)

Figure 2

NPL scores of genome-wide linkage analysis.

505 individuals with 52 affected (cases), 127 unaffected (controls) and 326 individuals with other phenotypes.

\section{Statistical methods}

To explore the phenotype $\times$ genotype relationship and locate the $\mathrm{AD}$ predisposing genes, we carried out a fivestage procedure. The first stage was designed to search the potential candidate regions by considering a genomewide linkage analysis using the STRP markers. GENEHUNTER [2] and SIMWALK2 [3] were employed to conduct multipoint nonparametric linkage (NPL) analysis, using the 'all' scoring function. Five evenly spaced positions scanning between markers were used. The allele frequencies were provided by GAW14 working group. A chromosome region with an NPL score greater than 3 was identified as "highly linked with $\mathrm{AD}$ ".
The second stage used denser SNP markers to confirm linkage results obtained in the first stage. On the basis of the NPL scores from the first stage, a candidate region was defined to be a segment in which all NPL scores exceeded 1 and the maximal NPL score exceeded 3 . In the candidate regions, SIMWALK2 [3] was carried out for multipoint linkage analyses using Illumina and Affymetrix SNP markers. The results were compared with those obtained from the first stage.

In the third stage, association analyses were conducted using SNPs to further narrow the candidate region. Transmission disequilibrium tests were performed by using PDT [4] and FBAT [5] for family data, and linkage disequilibrium tests (allele-based association test [6]) were used for case-control data. 
BMC Genetics 2005, 6:S30

Table I: Summary of results of transmission and linkage disequilibrium tests

\begin{tabular}{|c|c|c|c|c|c|}
\hline Chr. & SNP & Position & PDT & FBAT & Allele \\
\hline \multirow[t]{18}{*}{ I } & rsl906255 & 182.8 & & & 2.75 \\
\hline & tsc0073840 & 204.8 & & & 2.02 \\
\hline & tsc098209। & 212.1 & 2.41 & & \\
\hline & tscl I00744 & 213.3 & & & 2.49 \\
\hline & rs908857 & $2 \mid 4.1$ & 2.37 & & \\
\hline & tsc0054439 & 215.3 & & & 2.13 \\
\hline & $\operatorname{tsc}|5| 202 \mid$ & 219.3 & & & 2.29 \\
\hline & tsc004085 I & 219.6 & & & 2.42 \\
\hline & tsc0056649 & 224.8 & & & 2.60 \\
\hline & $\mathrm{tsc} 0576563$ & 225.6 & & & 2.25 \\
\hline & tscl001020 & 226.4 & & & 3.42 \\
\hline & tsc0059489 & 226.7 & & 2.88 & \\
\hline & tsc0990050 & 229.3 & & & 4.22 \\
\hline & tsc0998408 & 234.7 & 2.01 & & \\
\hline & $\mathrm{tsc} 0272628$ & 238.9 & & & 2.68 \\
\hline & tsc0832886 & 248.6 & & & 2.99 \\
\hline & rs94600I & 257.0 & 2.41 & & \\
\hline & tsc0046578 & 260.0 & & 2.99 & \\
\hline \multirow[t]{14}{*}{2} & $\operatorname{tsc} 1457260$ & 40.79 & & & 2.04 \\
\hline & tsc0275086 & 45.06 & 2.49 & & \\
\hline & rs2033654 & 47.60 & 2.30 & 2.09 & \\
\hline & tscl518799 & 55.32 & & 2.69 & \\
\hline & rs927087 & 56.10 & 2.25 & & \\
\hline & tsc0273405 & 56.65 & & & 2.81 \\
\hline & tsc07647/4 & 59.83 & 2.00 & & \\
\hline & rs726548 & 66.90 & & 2.00 & \\
\hline & tsc0788448 & 71.22 & & & 2.15 \\
\hline & tsc0043992 & 75.99 & 3.15 & & \\
\hline & tsc0270239 & 80.94 & & 2.17 & \\
\hline & rs20083I2 & 88.20 & & & 2.46 \\
\hline & rs977744 & 102.1 & & & 3.93 \\
\hline & tsc0794923 & 102.5 & 2.13 & & \\
\hline \multirow[t]{21}{*}{7} & tsc005039l & 5.21 & & & 2.02 \\
\hline & tscll I 0728 & 13.65 & & 2.02 & \\
\hline & tsc0847689 & |7.8| & & & 2.88 \\
\hline & tsc00644I9 & 18.04 & & & 2.60 \\
\hline & $\mathrm{tsc} 024773 \mathrm{I}$ & 20.41 & & 2.38 & \\
\hline & tscl061178 & 29.05 & & 2.08 & \\
\hline & rs957960 & 29.30 & & & 2.02 \\
\hline & $\mathrm{tsc} 0331830$ & 33.94 & & 2.15 & \\
\hline & $\mathrm{tsc} 0050450$ & 35.30 & & & 2.07 \\
\hline & rs798646 & 37.90 & & & 2.14 \\
\hline & tsc0593964 & 42.62 & 2.72 & 3.48 & \\
\hline & tsc0042959 & 44.49 & & 2.69 & \\
\hline & $\operatorname{tsc} 0051325$ & 47.56 & 2.17 & 2.24 & \\
\hline & tsc0054307 & 47.85 & 2.17 & 2.24 & \\
\hline & $\mathrm{tsc} 0462262$ & 61.4514 & & & 2.45 \\
\hline & $\operatorname{tsc}|24| 245$ & 62.6748 & 2.14 & & \\
\hline & tsc0893346 & 63.6850 & & & 2.37 \\
\hline & tsc0065973 & 70.5578 & & & 2.00 \\
\hline & $\mathrm{tsc} 0260324$ & 73.8253 & & & 2.13 \\
\hline & $\mathrm{tsc} 0525473$ & 75.0415 & & & 2.26 \\
\hline & $\operatorname{tsc} 1407569$ & 75.6400 & & & 4.75 \\
\hline
\end{tabular}


Table 2: Summary of results of haplotype analysis with covariates

\begin{tabular}{|c|c|c|c|c|}
\hline Study design & Chr & SNPs in haplotype & Significant haplotypes & OR $(95 \% \mathrm{Cl})$ \\
\hline Family-based & 1 & tsc0046578 - -tsc09383। 7 & $---b$ & --- \\
\hline Family-based & 2 & tsc0063067-tsc0059588-tsc0043992-tsc| $47350 \mathrm{I}^{\mathrm{a}}$ & 2222 & $0.65(0.45,0.93)$ \\
\hline Family-based & 7 & tsc00I87| 3-tsc00I87|2-tsc0593964a & III & $2.13(1.09,4.15)$ \\
\hline Case control & 1 & tsc0949090-tsc075535I-tsc0990050a & --- & --- \\
\hline \multirow[t]{2}{*}{ Case control } & 2 & rs977744a-tsc0794923 & 11 & $0.001(<0.001,0.207)$ \\
\hline & & & 12 & $0.009(0.00 I, 0.17 \mathrm{I})$ \\
\hline Case control & 7 & tsc0272090-tsc| 407569a & --- & --- \\
\hline
\end{tabular}

aAnchor marker

b.--, no significant haplotypes were identified.

In the fourth stage, anchor markers were selected on the basis of results from the third stage. HAPLOVIEW [7] was used to construct haplotype blocks and select tag SNPs in the region determined by anchors and nearby markers. Inferences on genotype-phenotype relationship were drawn by results obtaining from haplotype analysis using SIMWALK2 [3] for family data and PHASE2 [8] for casecontrol data.

In the fifth stage, the relationships between genotype, phenotype, and covariates underlying the complex alcoholism etiology was further explored. The individual haplotypes were inferred based on results obtained from SIMWALK2 [3] for family data and PHASE2 [8] for casecontrol data. The inferred individual haplotypes and important demographic variables, risk factors, and other phenotypes were modelled simultaneously with the explanatory variables in the regression models. For family-based analysis, the generalized estimating equation approach using the procedure GENMOD of the package SAS/STAT [9] was applied; for case control analysis, an unconditional logistic regression using the procedure LOGISTIC of the package SAS/STAT [9] was applied. The flow chart of statistical analyses is shown in Figure 1.

\section{Results}

A genome-wide multipoint linkage analysis for the 22 pairs of autosomal chromosomes based on the 315-STRP markers using GENEHUNTER [2] was conducted. Figure 2 (the green solid line) shows that NPL score $>3$ only occurs on chromosome 7 and the highest NPL score (3.54866) is located at D7S2846.

To reduce false-positives due to population heterogeneity, stratified analyses by selecting non-Black and non-White subpopulations from the whole population was conducted. Whole-genome linkage mapping with STRP markers was applied to these two subpopulations and yielded rather different results compared with the whole population. The results are shown in Figure 2. For the non-White population (the blue dashed line), no NPL score was found to be larger than 3 , which might be due to small sample size in this subpopulation. For the non-Black population (the orange dot-point line), the NPL scores for D1S225, D1S547, and D2S1356 are 3.91886, 4.10389, and 4.43759, respectively. Results obtained from GENEHUNTER [2] and SIMWALK2 [3] are quite consistent (results not shown).

In the second stage, we conducted SNPs linkage analysis to confirm the STRP linkage results of chromosomes 1, 2, and 7 found in the first stage. The three candidate regions determined by the mentioned criteria were D1S518D1S547, D2S320-D2S436, and D7S1790-D7S665. In these three candidate regions, the Linkage III Panel of SNPs of Illumina consists of 38,151, and 103 SNPs and the inter-marker distances are $0.99,0.53$, and $0.74 \mathrm{cM}$ in average. The GeneChip Mapping $10 \mathrm{~K}$ Array marker set of SNPs of Affymetrix consists of 113, 344, and 238 SNPs and the average distances between markers are $0.47,0.23$, and $0.30 \mathrm{cM}$. The results confirm the previous linkage results and find significant Illumina and Affymetrix SNPs with NPL scores $>3$ on chromosome 2 as shown in Figure 1 ; however, the NPL curves are not the same as the curve obtained from STRPs previously.

In the third stage, further fine mapping was pursued to narrow down the candidate regions using association tests. Based on family-based transmission disequilibrium tests (PDT [4] and FBAT [5]) and case-control linkage disequilibrium tests (allele-based test [6]), the SNPs associated with $\mathrm{AD}$ ( $p$-value $<0.01)$ without correcting multiple tests are shown in Table 1, where $p$-values are transformed by taking $-\log _{10}$.

In the fourth stage, we selected the most significant SNPs to be anchor markers based on Table 1 and preceded with finding haplotype blocks and tag SNPs in the region. Only the block closest to the anchor marker was used to conduct haplotype analysis without adjusting covariates. However, no significant haplotypes were found.

In the fifth stage, haplotype regression analyses considering three significant covariates (age, gender, and max 
drink) were conducted, which were selected in preliminary analysis. Results of adjusted odds ratio are summarized in Table 2. On chromosome 1, no significant haplotypes were found. On chromosome 2, haplotypes 11 and 12 constituted by SNPs rs 977744 and tsc0794923 yield ORs 0.001 and 0.009 and 95\% CIs $(<0.001,0.207)$ and $(0.001,0.171)$, respectively, and show strong protective effects; haplotype 2222, comprising SNPs tsc0063067, tsc0059588, tsc0043992, and tsc1473501 at gene NRXN1, yields an OR of 0.65 with $95 \%$ CI $(0.45$, 0.93). On chromosome 7, haplotype 111 from SNPs tsc0018713, tsc0018712, and tsc0593964 is a risk haplotype with an OR of 2.13 and corresponding 95\% CI (1.09, 4.15).

\section{Discussion}

In summary, some potential candidate regions on chromosomes 1, 2, and 7 linked with AD susceptibility loci were found. These findings are consistent with previous reports [10,11]. Moreover, association and haplotype analyses further narrowed the candidate region. On chromosome 2, a haplotype within the intronic region of gene NRXN1 related to polymorphic cell surface proteins was identified, as well as two strongly protective haplotypes in inter-gene regions. On chromosome 7 , one moderately risky haplotype in an inter-gene region was identified. These results should be useful to biologists for the advanced study of functional cloning.

The linkage scans based on three different marker sets were compared. The curves of NPL scores based on two SNP sets are quite similar; however, the SNP scans and STRP scan show somewhat inconsistent results on different chromosomes. On chromosome 2, SNP linkage scan confirms STRP scan and yields more and higher linkage signals in the same region. In other candidate regions, SNP scans fail to identify any important SNPs, probably due to their lower information content. We also compared the results from three association tests and found many different significant SNPs based on family-based and case control association tests. The differences were probably due to the different samples used in the analyses and information extracted from transmission and linkage disequilibrium tests.

Our five-stage gene mapping procedure is elaborate though incomplete. Other analytical strategies, such as quantitative trait analysis, will provide complementary information to further dissect the etiology of AD.

Abbreviations

AD: Alcohol dependence

COGA: Collaborative Study on the Genetics of Alcoholism
GAW: Genetic Analysis Workshop

NPL: Nonparametric linkage

SNP: Single-nucleotide polymorphism

STRP: Short tandem repeat polymorphism

\section{Authors' contributions}

$\mathrm{H}-\mathrm{CY}$ conceived the statistical analysis scheme, coordinated the project and drafted the manuscript. CSJF contributed to the discussion and preparation of the final manuscript. Other members carried out the data management, statistical analysis and technique assistance. All authors have approved the final manuscript.

\section{Acknowledgements}

We appreciate two anonymous reviewers' constructive comments. This work is partially supported by grants NSC 92-3II $2-\mathrm{B}-00 \mathrm{I}-014$ and 93IBMS2PP-C of Taiwan.

\section{References}

I. Almasy L, Borecki IB: Exploring genetic analysis of complex traits through the paradigm of alcohol dependence: summary of GAW I I contributions. Genet Epidemiol I999, I 7(SuppI I):SI-S24.

2. Kruglyak L, Daly MJ, Reeve-Daly MP, Lander ES: Parametric and nonparametric linkage analysis: a unified multipoint approach. Am J Hum Genet 1996, 58:1347-1363.

3. Sobel E, Lange K: Descent graphs in pedigree analysis: applications to haplotyping, location scores, and marker sharing statistics. Am J Hum Genet 1996, 58:1323-1337.

4. Martin ER, Monks SA, Warren LL, Kaplan NL: A test for linkage and association in general pedigree: the pedigree disequilibrium test. Am J Hum Genet 2000, 67:146-154.

5. Laird NM: Family based tests for associating haplotypes with general phenotype data: application to asthma genetics. Genet Epidemiol 2004, 26:6I-69.

6. SAS Institute Inc: SAS/Genetics User's Guide North Carolina; 2002.

7. Barrett JC, Fry B, Maller J, Daly MJ: Haploview: analysis and visualization of LD and haplotype map. Bioinformatics 2005, 21:263-265

8. Stephens M, Smith NJ, Donnelly P: A new statistical method for haplotype reconstruction from population data. Am J Hum Genet 200I, 68:978-989.

9. SAS Institute Inc: SAS/Stat User's Guide North Carolina; 1999.

10. Reich T, Edenberg HJ, Goate A, Williams JT, Rice JP, Van Eerdewegh $\mathrm{P}$, Foroud T, Hesselbrock V, Schuckit MA, Bucholz K, Porjesz B, Li TK, Conneally PM, Nurnberger JI Jr, Tischfield JA, Crowe RR, Cloninger CR, Wu W, Shears S, Carr K, Crose C, Willig C, Begleiter H: Genome-wide search for genes affecting the risk for alcohol dependence. Am J Med Genet 1998, 81:207-2I5.

II. Aragaki C, Quiaoit F, Hsu L, Zhao LP: Mapping alcoholism genes using linkage/linkage disequilibrium analysis. Genet Epidemiol 1999, 17(SuppI I):S43-S48. 